\title{
Progress and Challenges of Global High-Resolution Endoscopy
}

\author{
Sheena Bhushan ${ }^{1,2^{*}}$ D, Rebecca Richards-Kortum ${ }^{3}$ and Sharmila Anandasabapathy ${ }^{1,2}$ \\ ${ }^{1}$ Section of Gastroenterology and Hepatology, Department of Medicine, Baylor College of Medicine, USA \\ ${ }^{2}$ Baylor Global Health, Baylor College of Medicine, USA \\ ${ }^{3}$ Department of Bioengineering, Rice University, USA
}

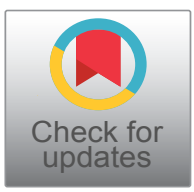

*Corresponding author: Sheena Bhushan, MD, Section of Gastroenterology and Hepatology, Department of Medicine, Baylor College of Medicine, Medical Center, 1 Baylor Plaza, Houston, TX 77030, USA, Tel: 713-798-8092

\begin{abstract}
In recent years, gastrointestinal endoscopy has seen an influx of high-resolution endoscopic technologies that are capable of providing optical biopsies of the epithelial surface in real time. Upcoming high-resolution endoscopy techniques are CLE, OCT, EC and HRME. Powered by automated diagnostic algorithms and more-cost effective alternatives, these high-resolution endoscopic technologies have the potential to not only facilitate real-time decision making, but also improve screening and surveillance of gastrointestinal cancers globally. This review discusses the most promising upcoming optical biopsy technologies and their clinical applications, along with the current trends, challenges and future directions.
\end{abstract}

\section{Introduction}

Recent advances in endoscopic technology have improved mucosal visualization, lesion detection and also allowed for real-time optical diagnosis. High definition and optical contrast imaging technologies such as narrowband imaging, iScan, blue laser imaging, and autofluorescence have been used as complementary, enhancement techniques for white light endoscopy. These technologies are used as "widefield imaging" techniques that help unmask or highlight inconspicuous mucosal abnormalities not visible to the human eye. While these techniques are invaluable as "red flag" techniques that highlight suspicious areas for further assessment with other imaging modalities, and are considered high resolution, they cannot yield cellular and subcellular imaging. Examples of some newer high-resolution endoscopic technologies include Confocal Laser Microendoscopy (CLE), Optical Coherence Tomography (OCT), Endocytoscopy (EC) and High Resolution Microendos- copy (HRME) (Figure 1). These technologies can image the gastrointestinal mucosa at a cellular and subcellular level and thus provide a higher degree of resolution as compared to the widefield imaging techniques. By providing subcellular imaging of the epithelial surface, these 'optical biopsy' technologies have the potential to revolutionize endoscopy and can offer a more targeted, efficient and cost-effective approach to endoscopic screening and surveillance. This review will focus on these emerging ultra-high-resolution endoscopy technologies and aims to provide a technical overview of the technologies, their clinical applications and also discuss the challenges associated with them.

\section{Technical Overview}

\section{Confocal laser endomicroscopy}

Confocal laser endomicroscopy (CLE) is an endoluminal imaging technique that enables real-time, high-resolution assessment of the gastrointestinal mucosa at a cellular and subcellular level. By providing in vivo "optical biopsies", it has expanded the current scope of standard flexible endoscopy significantly. The term "confocal" refers to the alignment of both illumination and collection systems in the same focal plane [1]. Fluorescence CLE uses the principle of excitation of a fluorophore with low-power laser light ( $488 \mathrm{~nm})$, followed by subsequent detection of the remitted fluorescent light through a conjugate pinhole [2]. Light emitted from a specific depth is refocused at the conjugate plane and passes through the pinhole confocal aperture. As a result, light emitted from above and below the plane of interest is not detected, and the point of illumination

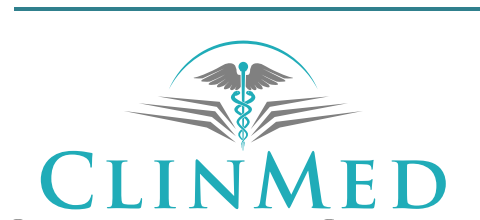

INTERNATIONAL LIBRARY

Citation: Bhushan S, Richards-Kortum R, Anandasabapathy S (2020) Progress and Challenges of Global High-Resolution Endoscopy. Int Arch Intern Med 4:024. doi.org/10.23937/2643-4466/1710024 Accepted: April 21, 2020: Published: April 23, 2020

Copyright: (c) 2020 Bhushan S, et al. This is an open-access article distributed under the terms of the Creative Commons Attribution License, which permits unrestricted use, distribution, and reproduction in any medium, provided the original author and source are credited. 


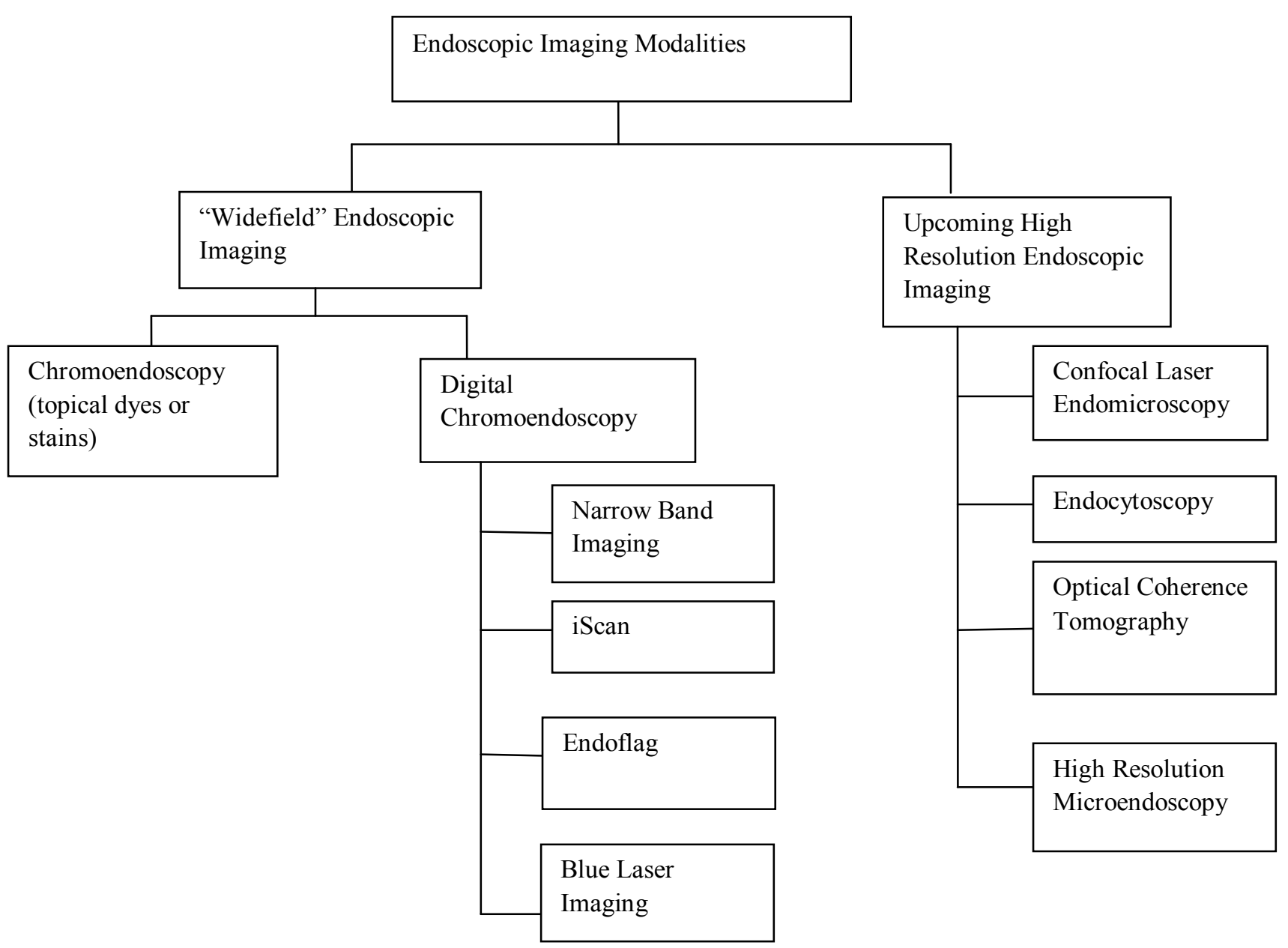

Figure 1: Classification of endoscopic imaging techniques.

coincides with the point of detection within the specimen, dramatically improving spatial resolution [3,4]. The emission of the fluorophore at the point of illumination is recorded to create a pixelated two-dimensional image of the tissue sample. Multiple points are analyzed, and the fluorescence emission is displayed as a gray scale-image. Three-dimensional (3-D) images can be created by using bench-top confocal microscopes. The high spatial resolution of CLE makes in vivo microscopic imaging of gastrointestinal mucosa possible. Currently, two CLE platforms are approved by the FDA: 1) Endoscope-based confocal laser endomicroscopy (eCLE); 2) Flexible fiber based confocal miniprobe.

Confocal laser endomicroscope (eCLE): While eCLE was developed and evaluated in multiple clinical studies, the device is not commercially available at present. The confocal laser endomicroscopy (Pentax, Tokyo, Japan), uses a miniaturized confocal scanner that is integrated into the distal end of a standard endoscope and allows the endoscopist to use white-light endoscopy and eCLE simultaneously [5]. The system consists of two additional buttons on the endoscope, that allow the endoscopist to control the depth of imaging relative to the surface of the tissue in a $7 \mu \mathrm{m}$ increments, to a maximum of $250 \mu \mathrm{m}$ [6]. eCLE has a field of view is $475 \times 475 \mu \mathrm{m}$ and a lateral resolution of $0.7 \mu \mathrm{m}[6,7]$.
The confocal images are collected at a scan rate of 0.8 frames/seconds ( $1024 \times 1024$ pixel) or 1.6 frames/second (1024 × 512 pixels) and displayed on a separate monitor next to the WLE image monitor $[6,7]$. Image stabilization is crucial to obtain high-quality images. While the eCLE can be used to examine luminal structures in the upper and lower gastrointestinal tracts, it is still quite large and cannot be used for biliary intraductal examinations [5].

Probe-based confocal laser endomicroscopy (pCLE): Alternately, a flexible Probe-based confocal laser endomicroscope ( $p C L E$ ) can also be used. The system (Cellvizio, Mauna Kea Technologies, Paris, France) uses a confocal microscope that is introduced as a miniprobe through the accessory channel of a standard endoscope [5]. The mini probe is exceptionally flexible, with a diameter of 0.9 to $0.255 \mathrm{~mm}$ and thus can be easily introduced through the accessory channel of virtually any endoscope (including a cholangioscope, bronchoscope) [5]. Both the light source and the laser scanning units are outside the human body, with the miniprobe acting as a passive conduit. The fiber probe consists of up to 30,000 optical fibers that transmit the $488 \mathrm{~nm}$ laser light and then also return the reflected fluorescent light to the distal micro-objective. Confocal images are collected at a rate of 12 frames per second, allowing in 
vivo imaging of capillary blood flow. Unlike eCLE it has a slightly lower resolution, a smaller field of view, and a fixed depth of imaging. Depending on the type of probe used, the field of view ranges from 240-600 um, the lateral resolution ranges from 1-3.5 um and the depth of imaging ranges from 0-100 um [5]. Cholangioflexprobe is a newer miniaturized pCLE with a diameter of $0.9 \mathrm{~mm}$, that is small enough to be introduced into cholanigoscopes and can be used to examine pancreatic and biliary structures [8]. A further miniaturized device called the needle-based CLE ( $\mathrm{nCLE}$ ) has a probe that can be passed through a 19G-FNA needle, thereby enabling endoscopic ultrasound-guided CLE of solid organs, lymph nodes, and cystic lesions [5]. The probes can be disinfected and reused up to 10-20 times, after which they require replacement [5].

Fluorescent contrast agents: Since CLE relies upon tissue fluorescence, exogenous fluorescence contrast agents are needed. The most commonly used contrast agent is IV fluorescein which is given to the patient intravenously. It allows visualization of cellular architecture by highlighting the vasculature, lamina propria and intracellular spaces of the tissue under examination [9]. Fluoresceins are nontoxic and excreted renally. They are sometimes associated with rare adverse events including hypotension without shock $(0.5 \%)$, nausea $(0.39 \%)$, injection site redness $(0.35 \%)$, diffuse self-limited rash (0.04\%), mild epigastric pain $(0.09 \%)$ and anaphylaxis (uncommon but has been reported) [10]. However, since flouresceins do not stain the nuclei, they cannot be used to assess nuclear-to-cytoplasmic ratio, used for diagnosis and grading intraepithelial neoplasia. Nuclear staining can be accomplished using topical contrast agents that are sprayed on to the mucosa directly. Acriflavine ( $0.05 \%$ in saline) accumulates within the nuclei and has been used in evaluation in several studies but theoretical concerns around mutagenicity have limited widespread adoption [11]. Cresyl violet $(0.13 \%$ in acetic acid) is another topical agent that causes cytoplasmic enrichment leading to a negative visualization of nuclear morphology [12].

Obtaining biopsies: The eCLE is integrated into a standard scope, freeing the accessory channel which is used to obtain biopsies. However, since PCLE systems occupy the accessory channels, biopsies can only be obtained after removing the probe. This limitation can be overcome by creating a dimple before removing the miniprobe, can help demarcate the area of interest [13].

\section{Endocytoscopy (EC)}

Endocytoscopy is another emerging endoscopic technology that allows real-time, high-resolution assessment of gastrointestinal mucosa at a cellular and sub-cellular level. It differs from CLE in that it uses the principle of white-light contact microscopy i.e., optical lenses are placed direct contact with the tissue to achieve high-level magnification [14]. Unlike CLE, there is no confocal plane, hence only the superficial layer of mucosa can be imaged. To visualize the nuclei and other cellular and subcellular structures, topical application of methylene blue $(0.5 \%$ to $1 \%)$ or a combination of methylene blue with crystal violet is used [15]. Mucus often hampers contrast penetration, staining and hence visualization of cell details; therefore, prior treatment with a mucolytic agent like $\mathrm{N}$-acetylcysteine is typically done [16]. After application of the stain, the tip of the endoscope is placed in direct contact with the mucosa, and the mucosal surface is scanned condensed with white light. The fixed-focus high-power optical lens then projects ultra-magnified images from a small mucosal sampling site (less than $0.5 \mathrm{~mm}$ in diameter) on to a Charged-Coupled Device (CCD). EC allows for visualization of several cytological and architectural features including, size and arrangement of cells, the size, and shape of nuclei, the nuclear-to-cytoplasmic ration and other atypia [15].

\section{Endocytoscope}

Two types of endocytoscopes (Olympus Instruments, Tokyo, Japan) are currently available: The first type is a probe-based endocytoscope, that consists of two flexible catheters that provide a magnification of 450-1125fold (on a 14-inch monitor) or 500-1400-fold (using a 19-inch monitor) $[17,18]$. Both probes can be passed through the working channels of standard endoscope and be placed in direct contact with tissue for imaging. To provide stability and minimize motion artifact, a soft plastic cup at the tip of the endoscope is generally used. The second and the only commercially available endocytoscope system is integrated within an endoscope [19]. In addition to having conventional optical magnification, it also provides and image magnification of 580 -fold on a 19-inch monitor. The tip of the endoscope is placed in direct contact with the tissue to obtain images.

\section{Optical Coherence Tomography (OCT) and Volu- metric Laser Endomicroscopy}

Optical Coherence Tomography (OCT) was originally used in ophthalmology, and more recently, there have been several studies using OCT in the gastrointestinal tract [20]. OCT is an exciting field of gastrointestinal imaging technology that relies on the backscattering of light to obtain cross-sectional images of tissue [21,22]. It is similar in principle to ultrasonography but uses light waves instead of sound waves to generate images [23]. OCT is performed using different probes that can be introduced through the accessory channel of a standard endoscope. A radial probe is used to create radial images whereas a linear probe is used to create linear images. Unlike endoscopic ultrasound, OCT can also be performed through air. Therefore, no tissue contact or coupling is usually required [24]. Near-infrared light $(700-1500 \mathrm{~nm})$ is applied to the tissue, and subsequent optical backscattering of light is measured using low coherence interferometry to create cross-sectional images [25]. Images are displayed at a rate of 4 frames/ 
second. Most OCT systems achieve an axial resolution of approximately 7-20 um [24]. Scattering of light in the tissues decreases the depth of scanning to $1-2 \mathrm{~mm}$ in the GI tract [26]. As a result, OCT is only able to image the mucosa and submucosa during endoscopy. The high resolution that OCT provides allows for visualization of mucosal glands, crypts, villi. However, cellular features like nuclear dysplasia cannot be appreciated [27].

More recently, a newer version OCT system (NvisionVLE) became commercially available, which can image with in the wall of the esophagus, using a balloon catheter that can be used through the scope and placed in the distal esophagus [28]. The VLE utilizes optical signal acquisition and processing methods to create high-resolution cross-sectional images of the esophageal wall. VLE, compared to the original OCT system can scan a wider area ( $6 \mathrm{~cm}$ circumferential segment) of the esophagus in real time, to create high resolution (7 um), 3D cross-sectional images while reaching a depth of $3 \mathrm{~mm}$ below the mucosa [28]. The Nvision VLE offers a volumetric view of approximately $10.000 \mathrm{~mm}^{2}$ and has a significantly improved imaging speed and resolution (25 times higher than endoscopic ultrasound) as compared to the first-generation OCT systems [29]. The cross-sectional view of the Nvision VLE can collect approximately 1200 cross-sectional images across the targeted $6 \mathrm{~cm}$, with an option to zoom in for closer examination [29].

Alternatively, the longitudinal view allows the endoscopist to examine the plane of the esophagus perpendicular to its cross-section, with over 4000 longitudinal images of the esophagus [29]. Both these views can be easily manipulated using a touch screen monitor or a hand controller. Other recent advancements to this system include the ability to perform laser marking, as well as a more recent "artificial intelligence" system. The Real-Time Targeting TM allows clinicians to make tissue laser marks that are visible under WLE, to facilitate identification and localization of areas for target biopsies [29]. The Intelligent Real-time Image Segmentation TM (IRIS) artificial intelligence software aids image review by segmenting and colorizing the three most common esophageal image features including hyperreflective surface, layering, and hypo-reflective structures in real time [30]. While the safety and effectiveness of this diagnostic software has not yet been evaluated, the Nvision VLE has the potential to enable a more thorough examination of the area of interest, which could improve biopsy targeting.

\section{High Resolution Microendoscope}

The High Resolution Microendoscope (HRME) is a novel, low cost $(<\$ 3600)$, portable, battery-operated endoscope that like CLE can provide real-time, high-resolution assessment of the gastrointestinal mucosa at a cellular and subcellular level [31]. While the device is not yet commercially available, extensive clinical valida- tion has been performed and reported. The main components of the HRME are a light source, a fiber-optic bundle, a microscope objective lens, and a CCD camera. The HRME uses a 1-mm diameter flexible fiber-optic probe that is introduced through the accessory channel of a standard gastroscope or colonoscope and pressed perpendicularly against the mucosa, to yield histopathology-like images [31]. Similar to CLE, the HRME uses a fluorescent topical contrast agent. The most commonly used contrast agent is Proflavine Hemisulfate $(0.05 \%)$ [32]. Proflavine has peak absorption and emission wavelengths of $455 \mathrm{~nm}$ and 515 respectively [31]. A light-emitting diode provides excitation at $445 \mathrm{~nm}$, which is delivered through the probe to the mucosal surface. Excitation light is reflected at a $475 \mathrm{~nm}$ dichroic mirror to form an image at the back aperture of a 10 $\times 0.25$ NA infinity-corrected objective lens. The bundle collects fluorescent emission from the sample, transmitted through the dichroic mirror and emission filter, then imaged onto a CCD camera by a tube lens. Images are transferred from the camera to a PC for real-time display at 12 frames/second [33]. The images provide clear delineation of cellular features like nuclear size, crowding, and pleomorphism. The images are displayed at 12 frames/second ( $\times 400$ magnification). The spatial resolution of the probe is $4.4 \mu \mathrm{m}$ and the field-of-view is $720 \mu \mathrm{m}$ in diameter [32]. The probe can be easily disinfected and reused up to 75 times before it needs repolishing. Repolishing only takes minutes and restores the probe to its optimum performance levels.

\section{Obtaining biopsies}

Since the HRME probe occupies the accessory channel of the endoscope, biopsies can only be obtained after the probe is removed. Before removing the probe, it is often helpful to create a dimple that helps demarcate the area of interest making it easier to obtain a biopsy [34].

\section{Clinical Applications}

The high-resolution endoscopic modalities mentioned above (Table 1) can be used to facilitate early detection and treatment of neoplastic lesions in the gastrointestinal tract. In the next section of this review, we discuss the clinical applications and efficacy of these high-resolution endoscopy techniques for Barrett's Esophagus, Gastric Cancer, Colorectal Cancer, and Ulcerative Colitis.

\section{Barrett's esophagus (BE)}

Barrett's esophagus (BE) is widely prevalent in the West and is a known precursor lesion of Esophageal Adenocarcinoma. Endoscopic surveillance is done to enable early detection of neoplastic lesions including High-Grade Dysplasia (HGD) and Intramucosal Cancer (ICM). The current guidelines call for examination with white-light endoscopy with random four-quadrant biopsies every $2 \mathrm{~cm}$ [35]. However, this method is inef- 
Table 1: High-resolution endoscopy technologies.

\begin{tabular}{|l|l|l|l|l|l|l|}
\hline System & $\begin{array}{l}\text { Excitation } \\
\text { wavelength }\end{array}$ & $\begin{array}{l}\text { Lateral } \\
\text { resolution }\end{array}$ & Frame rate & Field-of-view & $\begin{array}{l}\text { Imaging } \\
\text { depth }\end{array}$ & Cost \\
\hline $\begin{array}{l}\text { Probe confocal } \\
\text { (pCLE) }\end{array}$ & $488 \mathrm{~nm}$ & $1.0-3.0 \mu \mathrm{m}$ & 12 frames/sec & $0.05-0.28 \mathrm{~mm}^{2}$ & $60-100 \mu \mathrm{m}$ & $\$ 150000$ \\
\hline $\begin{array}{l}\text { Endoscope } \\
\text { confocal (eCLE) }\end{array}$ & $488 \mathrm{~nm}$ & $0.7 \mu \mathrm{m}$ & 1.2 frames/sec & $0.23 \mathrm{~mm}^{2}$ & $0-250 \mu \mathrm{m}$ & $\$ 250000$ \\
\hline $\begin{array}{l}\text { Endoscope } \\
\text { Endocytoscopy }\end{array}$ & $400-700 \mathrm{~nm}$ & $1.7 \mu \mathrm{m}$ & 30 frames/sec & $0.4 \mathrm{~mm}^{2}$ & $0-50 \mu \mathrm{m}$ & $\mathrm{N} / \mathrm{A}$ \\
\hline $\begin{array}{l}\text { Optical coherence } \\
\text { tomography/VLE }\end{array}$ & $700-1500 \mathrm{~nm}$ & $7-10 \mu \mathrm{m}$ & 4 frames/sec & $5-6 \mathrm{~mm}^{2}$ & $2-3 \mathrm{~mm}$ & $\$ 35000-$ \\
\hline $\begin{array}{l}\text { High resolution } \\
\text { microendoscopy }\end{array}$ & $455 \mathrm{~nm}$ & $4.4 \mu \mathrm{m}$ & 12 frames/sec & $0.41 \mathrm{~mm}^{2}$ & $50 \mu \mathrm{m}$ & $<\$ 3600$ \\
\hline
\end{tabular}

*Not currently commercially available.

ficient and has missed neoplasia in up to $57 \%$ of cases [36,37]. High-resolution endoscopic imaging techniques can increase the diagnostic yield for neoplasia and also reduce unnecessary biopsies of non-neoplastic tissue. The American Society for Gastrointestinal Endoscopy (ASGE) proposed the following criteria to determine whether advanced imaging techniques with targeted biopsies can replace the current standard of care. It was recommended that in order to replace the current standard of care an imaging technology must show to have a per-patient sensitivity of $\geq 90 \%$, a specificity of $\geq 80 \%$, Negative Predictive Value (NPV) of $\geq 98 \%$ for detecting HGD as compared to the current standard [38].

\section{Colorectal cancer}

Colorectal Cancer (CRC) accounts for approximately $8 \%$ of all cancer deaths in the United States [39]. Early detection of colorectal cancer is a crucial strategy to reduce mortality associated with Colorectal Cancer (CRC). Differentiating high-risk polyps from benign lesions is critical to improve survival, reduce pathology costs, and decrease risks associated with unnecessary procedures. Using high-resolution endoscopy imaging that provides in vivo histology may enhance our ability to better select polyps for resection. In vivo diagnosis of early neoplastic changes can be made possible owing to these optical biopsy technologies.

\section{Clinical Applications of Confocal Laser Endomi- croscopy}

While there are no official guidelines for incorporating CLE in endoscopic surveillance yet, they are generally used to increase diagnostic yield during endoscopic surveillance. This is achieved increasing target biopsies of abnormal tissue while sparing biopsies of normal tissue. CLE has been studied for use in the upper and lower GI tract, the hepatobiliary system, and even the pancreas. In the next section of this review, we will discuss these studies more in detail.

\section{CLE and Barrett's esophagus}

CLE provides real-time "optical biopsies" of the ex- amined GI mucosa and thus can be used for the early detection neoplastic lesions in Barrett's esophagus. There have been several studies that have studied the use of CLE in BE with high sensitivity and specificity.

In a multicenter, randomized controlled trial conducted by Canto, et al. 192 patients with BE were assigned to either undergo High-Definition White-Light Endoscopy (HDWLE) alone with random biopsies or high-definition white-light endoscopy and eCLE with Targeted Biopsies (TB) [40]. Real-time diagnosis and treatment plans were recorded after HDWLE in both groups, and after eCLE in the second group. Blinded expert pathology diagnosis was the reference standard. Results showed that diagnostic yield and sensitivity for neoplasia detection was significantly higher in the group that underwent WLE + CLE with target biopsies as compared to the group that underwent WLE with random biopsies but with comparable accuracy. The combination of HDWLE + eCLE + TB tripled the diagnostic yield for neoplasia ( $22 \%$ vs. $6 \%$ ) and would have negated the need for biopsy in $65 \%$ of patients. Adding eCLE to HDWLE increased the sensitivity of detecting neoplasia from $40 \%$ to $96 \%$, while keeping specificity the same. Additionally, the use of eCLE altered the treatment plan in $36 \%$ patients. This study demonstrated that adding eCLE to HDWLE improves real-time endoscopic diagnosis of Barrett's esophagus related neoplasia and TB of abnormal areas decreases unnecessary biopsies. By enabling in vivo decision making, it also alters the course of management significantly.

In another multicenter, randomized control trial conducted by Sharma, et al., the authors compared the sensitivity and specificity of HDWLE + pCLE with HD-WLE alone for detection of BE associated neoplasia [41]. A total of 101 consecutive patients with $\mathrm{BE}$ undergoing either surveillance or treatment were enrolled, and all were examined by HD-WLE, NBI, and pCLE. Patients were randomly assigned to 2 endoscopy sequences (HDWLE followed by NBI and NBI followed by HD_WLE), and 4-quadrant random locations were recorded. These 4-quadrant locations were examined pCLE, and a diag- 
nosis of neoplastic or non-neoplastic was made in real time. Following imaging and recording biopsies were taken and sent for histopathology. Results showed that the sensitivity and specificity for pCLE or HD-WLE were $68.3 \%$ and $87.8 \%$ as compared to $34.2 \%$ and $92.7 \%$ for HD-WLE alone. The sensitivity and specificity for HDWLE or NBI were $45 \%$ and $88 \%$ respectively compared with $75 \%$ and $84 \%$ for HD-WLE, NBI, or pCLE. Additionally, the use of pCLE in combination with HD-WLE and NBI enabled the detection of 2 and 1 additional neoplastic cases compared with HD-WLE and HD-WLE or NBI.

\section{CLE and Esophageal squamous cell carcinoma}

Since CLE provides an Optical biopsy of the mucosal lesions, it also very useful for diagnosing Esophageal Squamous cell Cancer (ESCC). In a study conducted by Pech, et al. the diagnostic potential of CLE for diagnosing ESCC was evaluated in 21 consecutive patients with suspected lesions [42]. Following Lugol's Chromoendoscopy, the unstained areas were assessed using CLE, and a diagnosis was made in real time. Subsequently, biopsies were acquired from all unstained areas and sent for histopathology. A total of 43 lesions were imaged using CLE images, which were reviewed by two endoscopists, who were blinded to the histology and endoscopic appearance. Results demonstrated that CLE had an overall accuracy of $95 \%$, a sensitivity of $100 \%$ and a specificity of $87 \%$ for detecting early ESCC. Thus CLE, has the potential to provide an immediate real-time diagnosis with a high degree of accuracy.

In another study Huang, et al. evaluated the diagnostic utility of Lugol's chromoendoscopy guided-CLE in detection of superficial squamous cell carcinoma [43]. A total of 52 high-risk patients were enrolled and underwent Lugol's chromoendoscopy-guided CLE with subsequent recording of in vivo histological diagnosis. This was followed by the acquisition of Lugol's Chromoendoscopy guided biopsies by a second endoscopist, who was blinded to the CLE diagnosis. Using biopsy results, en-bloc resection of neoplastic areas was done. When histopathology from the en-bloc specimens was used as gold standard, compared to Lugol's guided biopsies, Lugol's-guided CLE exhibited a superior sensitivity (95.7\% vs. $82 \%)$, specificity (90\% vs. $70 \%)$, NPV $(81.8 \%$ vs. $46.7 \%)$ and accuracy (97.8\% vs. $92.7 \%)$ in diagnosing superficial Esophageal Squamous Cell Carcinoma.

\section{CLE and Gastric cancer}

Several studies have suggested that CLE can be a useful tool for evaluating gastric cancer and diagnostic accuracy of greater than $90 \%$ has been reported. Kitabatake, et al. conducted a study with 27 patients and obtained CLE images from 52 examined sites that were subsequently interpreted by two pathologists [44]. The sensitivity of CLE for gastric neoplasia was $82-91 \%$, and the specificity was $98 \%$. However, CLE was associated with a high failure rate (40\%) for image acquisition, which may limit the value of CLE for detecting gastric neoplasia. In another study by Zhang, et al. 521 sites from 137 patients were evaluated by CLE. When a previously developed pit pattern was present, CLE had a sensitivity of $90 \%$ and a specificity of $99 \%$ for diagnosing gastric cancer. Lastly, Li, et al. compared CLE with White Light endoscopy on 1572 patients undergoing upper endoscopy. Results showed that the sensitivity of CLE for detecting high-grade dysplasia or early gastric cancer was higher than WLE ( $89 \%$ vs. $72 \%)$. The specificity of CLE was also higher than WLE (99\% vs. 95\%).

CLE can also be used to reduce unnecessary biopsies as was demonstrated by the following two studies. In the first study by Jeon, et al. 31 patients with previously diagnosed 35 gastric epithelial neoplastic sites were scheduled to undergo ESD. CLE was used to image target lesions in vivo in all these patients [45]. The overall accuracy of CLE for diagnosing gastric adenocarcinoma was compared with post-ESD histopathology and was considerably higher than conventional endoscopic biopsy (94.2\% vs. $85.7 \%)$. Secondly, Bok, et al. conducted a prospective study in 46 patients with 54 gastric epithelial neoplastic sites, who were previously diagnosed by endoscopic biopsy and scheduled to undergo ESD [46]. pCLE was performed in vivo, ex vivo and then compared with the post-ESD histopathology. Overall accuracy for pCLE was higher than endoscopic biopsy $(91.7 \%$ vs. $85.2 \%)$. The combined accuracy was even higher (98.1\%).

\section{CLE and Colon polyps}

Several studies have demonstrated the utility of CLE for colon polyps, which we will describe below. As a diagnostic tool, CLE was found useful for differentiating between non-neoplastic (hyperplastic) and neoplastic tissue (adenomas and carcinomas) in the colon. Shahid, et al. evaluated the use of pCLE as a diagnostic tool in a study where they compared the sensitivity, specificity, and accuracy of pCLE to NBI in 65 patients, who underwent high definition colonoscopy first with NBI, followed by pCLE [47]. The confocal videos were subsequently analyzed offline and classified as neoplastic or non-neoplastic while being blinded to endoscopic characteristics and histopathology. A total of 130 polys were assessed and compared to NBI, pCLE demonstrated higher sensitivity ( $86 \%$ vs. $64 \%$ ) but similar accuracy (82\% vs. $79 \%$ ) and a lower specificity (78\% vs. 92\%) for diagnosing neoplastic polyps. When NBI and pCLE were used in combination, the accuracy approached that of histopathology. The same group also used CLE to detect residual tumor left behind following endoscopic management [48]. 92 patients (129 EMR scars) undergoing evaluation of prior EMR sites were enrolled and were assessed using VCE (NBI/FICE) and pCLE. Confocal videos of the scars were obtained in real time and were also analyzed offline, blinded to endoscopic characteristics and histopathology. Finally, biopsy confirmation or re- 
peat EMR was performed in all cases. Compared to VCE, pCLE had a superior sensitivity ( $97 \%$ vs. $72 \%$ ), accuracy ( $81 \%$ vs. $77 \%$ ), and similar specificity for detecting residual neoplasia. For scars where VCE and pCLE agreed on the diagnosis, the accuracy approached that of histopathology. Therefore, pCLE may be useful to identify and resect residual tumors in the same endoscopic session, eliminating the need for histopathology or unnecessary repeat procedures.

\section{Summary}

Thus, CLE can be used to confirm and optimize endoscopic diagnosis by decreasing unnecessary biopsies, interventions, and the cost, risks associated with them. Most studies demonstrate a good interobserver agreement for interpretation of CLE images (kappa = 0.6-0.8). However, interpreting CLE images requires considerable experience and training, thereby limiting its use among inexperienced gastroenterologists. Apart from the Miami classification for $\mathrm{pCLE}$, no international recommendations and guidelines are currently in place for other CLE systems [49]. The high costs associated with CLE systems (<175.000) also limits its adoption. Lastly, even though side effects to contrast agents are very rare, they have been reported (Table 2).

\section{Clinical Applications of Endocytoscopy}

Several studies have evaluated the use of Endocytoscopy in BE surveillance, Colon cancer screening, gastric cancer, and even in $\mathrm{H}$. pylori visualization. EC has shown to have a specificity. In the next section of this review, we will discuss those studies in more details.

\section{EC and Barrett's esophagus}

Studies have shown that Endocytoscopy has a sensitivity of 80 to 90 percent for detecting neoplasia in the squamous esophagus. However, the reported accuracy has been variable. For example, Pohl, et al. evaluated the ability of EC in diagnosing dysplasia in 16 patients undergoing surveillance for BE [50]. EC images were obtained using the $x 450$ and x1125 magnification lens, and subsequently, biopsies were taken sent for histopathology. A pathologist and gastroenterologist, both of whom were blinded to the histopathology results reviewed the images. Only $51 \%$ and $21 \%$ could be obtained at $x 450$ and $x 1125$ magnification respectively. At the $x 450$ magnification, EC had a sensitivity of $50 \%$ and a specificity of $67 \%$ for diagnosing BE. The sensitiv- ity decreased to $43 \%$, and the specificity increased to $85 \%$ when the $x 1125$ magnification was used. The study concluded that without visible evidence, EC lacked the image quality to be of any additional benefit.

In another example Eberl, et al. assessed the utility of EC for prediction non-neoplastic and neoplastic lesions in 25 patients with esophageal lesions [51]. Results showed that EC had a sensitivity of $81 \%$ and a specificity of $100 \%$ for diagnosing pathological esophageal lesions. The sensitivity increased to $91 \%$ when the X1100 magnification probe was used and decreased to $77 \%$ when the $x 450$ magnification lens was used.

By contrast, in a study by Inoue, et al. 28 patients with esophageal lesions that had previously been detected by regular/ + narrow band imaging were evaluated using EC [52]. Biopsies were also taken, and the images were compared with the histopathological gold standards. The authors were able to obtain clear images in all patients, and EC has a sensitivity of $88 \%$ and specificity of $91 \%$ for detecting malignancy.

\section{EC and Gastric cancer}

Kaise, et al. evaluated the use of in vivo EC in 82 patients with gastric lesions including early cancers (23), adenomas (10) and non-neoplastic lesions (49) [53]. Only $88 \%$ of these lesions could be imaged by EC because of poor staining due to thick mucus. Results showed that a finding of high-grade dysplasia had high sensitivity (86\%), specificity (100\%), PPV (100\%) and NPV (100\%) for diagnosing gastric cancer. This study concluded that in vivo EC imaging was reliable with high accuracy for diagnosing gastric cancer.

\section{EC and Colon cancer}

A small number of studies have evaluated the use of EC in diagnosing colon lesions, and the results seem quite promising. For example, Sasajima, et al. conducted a prospective study where they evaluated the accuracy of EC in 75 colonic lesions (59 neoplastic lesions, 16 non-neoplastic lesions) [19]. EC had a sensitivity and specificity of $100 \%$ in distinguishing neoplastic from non-neoplastic lesions. Of the 59 neoplastic lesions, $58 / 59$ were correctly identified as neoplastic, and 1/9 lesions was misdiagnosed as being neoplastic. The overall accuracy of EC was 93\%. This study demonstrated that $\mathrm{EC}$ has the potential to provide in vivo histological images with high sensitivity and accuracy.

Table 2: CLE performance.

\begin{tabular}{|l|l|l|l|l|l|l|}
\hline Reference & Year & Pathology & Total no. patients/lesions & Sensitivity & Specificity & Accuracy \\
\hline Canto, et al. [40] & 2014 & EAC & $\begin{array}{l}192 \text { patients; } 978 \text { biopsy } \\
\text { specimens }\end{array}$ & $96 \%$ & $92 \%$ & $93 \%$ \\
\hline Sharma, et al. [41] & 2011 & EAC & 101 patients & $68.30 \%$ & $87.80 \%$ & \\
\hline Pech, et al. [42] & 2008 & ESCC & 27 patients & $100 \%$ & $87 \%$ & $95 \%$ \\
\hline Huang, et al. [43] & 2015 & ESCC & 52 patients & $95.70 \%$ & $90 \%$ & $97.80 \%$ \\
\hline Kitabatake, et al. [44] & 2006 & Gastric cancer & 27 patients; 57 sites & $82-91 \%$ & $98 \%$ & \\
\hline
\end{tabular}


In another study, Mori, et al. demonstrated non-inferiority of EC compared with standard histopathology for colorectal neoplasms [54]. Patients with colorectal lesions $>5 \mathrm{~mm}$ were randomly assigned to an EC group and a standard biopsy group. An endoscopist and pathologist assessed the EC images and conventional biopsies respectively and gave a diagnosis. Using the final histopathology from the resected specimen, diagnostic accuracies were calculated. In total, 102 lesions in the EC group and 101 lesions in the standard biopsy group were taken into account. The diagnostic accuracy of EC was found to be non-inferior to standard biopsy $(94 \%$ vs. $96 \%)$.

More recently, Computer-aided Endocytoscopy has been used to assist with the diagnosis of colorectal cancer. Takeda, et al. evaluated 200 images of colonic lesions with Computer-aided Endocytoscopy and results showed a sensitivity of $89.4 \%$, a specificity of $98.9 \%$ in diagnosing invasive cancerous polyps [55].

\section{Clinical Applications of OCT and VLE}

\section{OCT and Barrett's esophagus}

OCT has been used in BE for surveillance of high-risk patients and to guide the selection of treatments likes ablation, EMR for BE related neoplasia and to detect "residual" Barrett's epithelium post-RFA. The earlier studies focused on identifying and describing key features indicative of including loss of layered architecture, change in surface signal intensity, presence of irregular glands in the epithelium [56-58].

Newer studies have also demonstrated the safety and feasibility of the second generation VLE system for the detection of dysplasia. In a prospective, observational study conducted by Smith, et al., 18 medical centers across the United States participated in completing a 1000-patient registry which aimed to assess the clinical utility of VLE for management of BE [59]. The NvisionVLE imaging system was used to identify areas of concern following which the investigators completed a short questionnaire regarding how VLE impacted each case. Results showed that in patients with previously diagnosed or suspected BE, VLE was used to identify areas of concern not visible under WLE in 59\% of the procedure. Additionally, VLE was also used to guide biopsy acquisition in $71 \%$ and treatment in $54 \%$ of the procedures. Overall, VLE improved the diagnostic yield by $55 \%$ beyond the current standard practice. In patients with no visible lesions on other imaging modalities and with no prior history of $\mathrm{BE}$, as compared to random biopsies VLE-guided biopsies improved neoplasia detection by $700 \%$. WLE and VLE, when used together provided an NPV of $100 \%$ for neoplasia in post-treatment population. Since VLE can image the mucosa, submuco$\mathrm{sa}$, and the muscularis propria, it helps the endoscopist with a more comprehensive evaluation as compared to WLE, NBI, and CLE that only image the superficial epi- thelium. Although this study had several limitations including lack of a control group, lack of histopathological interpretation of specimens, the results look promising especially in scenarios where no neoplasia is identified by other imaging modalities (clean scan). Additionally, this study was performed without the incorporation of a laser marking system, which has shown to significantly improve diagnostic yield in $\mathrm{BE}$ compared to the current standard Seattle Protocol [60].

More recently, computer-based diagnostic algorithms were developed that showed promising results for automated detection of BE neoplasia. Leggett, et al. compared the diagnostic performance pCLE and VLE for detecting dysplasia in ex vivo samples from 27 patients with $B E$ undergoing surveillance [28]. A total of 50 EMRs were imaged using pCLE and VLE and classified into neoplastic and non-neoplastic based on histology. The images were then analyzed and rated by 3 gastroenterologists with prior training in the established diagnostic criteria for both imaging systems. Additionally, the group also developed a novel VLE diagnostic algorithm to detect dysplasia based on VLE data from 27. Compared to the $\mathrm{PCLE}$, the VLE (using the previously established diagnostic criteria OCT-SI) had a sensitivity of $70 \%$ vs. $76 \%$, a specificity of $60 \%$ vs. $79 \%$ and an accuracy of $67 \%$ vs. $77 \%$. The novel diagnostic algorithm was reported to achieve a sensitivity of $86 \%$, a specificity of $88 \%$ and an accuracy of $87 \%$ for detecting Barrett's neoplasia with almost perfect interobserver agreement (kappa $=0.86$ ). Although this study was performed on ex vivo samples, with further refinement, the diagnostic algorithm has the potential to serve as a guide for in vivo real-time interpretation in the future.

Combined imaging and marking methods have been used in the past to achieve a more precise coregistration between OCT images and histology. VLE's new laser marking system makes direct in vivo marking of tissue possible. The visibility and locational accuracy of new laser marking technology was tested in vivo by Swager, et al. in 16 patients with BE. A variety of tissue types were images including normal epithelium, BE epithelium, LGD, HGD, EAC [60]. Results demonstrated high visibility of VLE laser marks under WLE (100\% in BE mucosa, $97 \%$ in squamous mucosa, $92 \%$ in gastric mucosa). Additionally, $100 \%$ of suspicious regions on OCT were successfully marked by laser marks, which allowed for targeted biopsies or EMR at targeted areas. By incorporating laser-guided targeted biopsies and EMRs, this technology has the potential to improve diagnostic yield and allow for a more accurate image and tissue correlation.

\section{OCT and Squamous cell carcinoma}

Studies have used OCT in the preoperative staging of Esophageal Squamous Cell Carcinoma (ESCC) to help guide treatment strategies. For example, Hatta, et al. evaluated the usefulness of in vivo OCT for the staging of superficial ESCC, in 62 patients with a histological di- 
agnosis of superficial esophageal squamous cell carcinoma [61]. OCT findings from the first 16 patients were used to classify the depth of invasion into three categories including 1) Epithelium; 2) Muscularis mucosa, and 3) Submucosa. The criteria were subsequently evaluated in the remaining 46 patients, and the results of OCT were compared with the gold-standard histopathology of resected specimens. The overall accuracy of OCT was $92.7 \%$. The accuracy of OCT for determining the involvement of the epithelium, muscularis mucosa and submucosa was $94.9 \%, 85 \%$, and $90.9 \%$ respectively.

In another study conducted later by the same group, OCT was compared with EUS for tumor staging of superficial esophageal squamous cell carcinoma in 123 patients with 131 superficial ESCC lesions [62]. An expert took representative images of all patients by using both OCT and EUS and reported the tumor staging. Another blinded expert reviewed the recorded images and reported staging independently. OCT was accurately able to identify $90 \%(118 / 131)$ of SESCC, over staged $4 \%(5 / 131)$ of tumors and under staged $6 \%(8 / 131)$ of the tumors. For the tumors that were limited to the epithelium, OCT was able to correctly stage $95 \%$ (88/93), which was considerably higher than that of EUS (81\%). For the tumors that involved the muscularis mucosa and submucosa, OCT was only able to correctly stage $79 \%(30 / 39)$.

Thus far, there have been no extensive studies that have incorporated the use of Nvision VLE. In a recent retrospective study by Trindade, et al. Nvision VLE system was used to stage superficial squamous cell carcinoma in 17 patients with intraepithelial neoplasia, T1a disease, T1b, T2 disease and T3 disease [63]. VLE scans were compared to gold standard histology for diseases T1b and greater. Results showed that VLE was able to distinguish superficial disease (limited to the lamina propria) and deeper disease in all cases. Although prospective studies are needed to confirm these findings, VLE has to potential to determine the extent of the disease in real time, which can alter management significantly (ESD vs. RFA).

\section{OCT of the colon and small bowel}

OCT enables the endoscopist to appreciate the full thickness of the colonic walls and can be useful in identifying neoplastic polyps. Adenomas are characterized by the presence of large glands in the superficial mucosa, mucosal cysts and an uneven surface, whereas Adenocarcinoma is characterized by a complete loss of mucosal architecture [64]. Pfau, et al. used real-time OCT to differentiate between adenomas, hyperplastic polyps, and normal colon tissue in 24 patient patients (44 polyps) [65]. Adenomatous polyps were characterized by a decrease in scattering intensity and the absence of a structured crypt. Hyperplastic polyps had an organized crypt pattern and the scattering intensity was similar to that of normal tissue.
Trindade, et al. used the Nvision VLE system in a case to evaluate a rectal polyp near the dentate line [66]. VLE showed normal tissue underneath the polyp, thereby altering the management from full-thickness resection to EMR.

\section{Summary}

OCT can provide high-resolution, wide area, subsurface imaging, and thus has the potential to improve diagnostic yield and efficiency of treatments. As with other imaging techniques, image interpretation still poses a challenge. The introduction of diagnostic algorithms is a possible solution, and with further advancements, real-time automated diagnosis of lesions with OCT may be probable.

\section{Clinical Applications of HRME}

\section{HRME and Barrett's esophagus}

Muldoon, et al. were the first to used HRME for surveillance of patients with $B E$ [32]. In the study, 9 patients who had previously been diagnosed with $B E$ underwent $E M R$. The resected tissue sample was imaged using the HRME, and the images were interpreted as being neoplastic or non-neoplastic by two gastroenterologists and two pathologists. HRME has a sensitivity of $87 \%$ and specificity of $61 \%$ in diagnosing neoplastic tissue when compared to gold-standard histopathology.

In another study by Vila, et al. HRME was evaluated for its accuracy and inter-rater reliability in diagnosing BE neoplasia [33]. HRME images were obtained from 28 consecutive patients undergoing endoscopic surveillance for HRME. 20 gastroenterologists with no prior microendoscopic experience and 3 experts microendoscopists (> 50 cases) then interpreted these images. HRME was found to have a sensitivity, specificity, NPV of $90 \%, 82 \%$, and $94 \%$ respectively for identification of neoplastic BE as compared with histology. Interobserver agreement was found to be moderate (0.56). No differences in accuracy were observed among experts and novices.

In the third study, Lee, et al. compared standard WLE with random biopsies with WLE + HRME with both targeted and random optical biopsies in 49 patients [67]. WLE + HRME had a higher diagnostic yield $(37.5 \%$ vs. $1.71 \%)$, a higher sensitivity ( $100 \%$ vs. $50 \%)$ and a comparable specificity ( $94.9 \%$ vs. $96.3 \%$ ) for diagnosing neoplasia as compared to WLE alone. Additionally, it was seen that with the use of HRME, $92.2 \%$ of individual biopsies could have been spared, with $71 \%$ of patients not requiring any biopsies at all.

\section{HRME and Esophageal squamous cell carcinoma}

In a prospective international trial conducted by Protano, et al. 147 consecutive high-risk patients undergoing endoscopic screening or surveillance were enrolled from 4 centers and underwent WLE followed by LCE and HRME [34]. A total of 7 endoscopists (3 microendosco- 
py experts, four novices) performed the procedure and interpreted the images which were compared with the gold-standard histology. Using a per-biopsy analysis HRME guided LCE vs. LCE had a sensitivity of 91 vs. $96 \%$, a specificity if $88 \%$ vs. $44 \%$, PPV of $45 \%$ vs. $22 \%$, NPV of $98 \%$ vs. $98 \%$ and an accuracy of $90 \%$ vs. $57 \%$ for diagnosing ESCC. By using per-patient analysis, HRME-guided LCE vs. LCE has a sensitivity of $95 \%$ vs. $100 \%$, a specificity of $79 \%$ vs. $29 \%$ and an accuracy of $83 \%$ vs. $47 \%$. With the use of HRME 136 biopsies could have been negated, with 55 patients not requiring any biopsies at all.

In another study Shin, et al. used the HRME to take images from 177 patients, which were used to develop an algorithm based on qualitative features to identify high-grade dysplasia or ESCC [68]. Mean nuclear area was used to classify images as either neoplastic or non-neoplastic and had a high specificity of $92-97 \%$ when compared to histology. The same group later developed and evaluated fully automated, real-time analysis software. The algorithm was implemented in a tablet-based HRME and used to analyze images from 3 high-risk patients. In a post-hoc quantitative analysis, the algorithm has a sensitivity of $95 \%$ and a specificity of 91\% for diagnosing neoplasia.

The same group also evaluated the accuracy of the software and its effect on the accuracy of post-hoc HRME image interpretation among endoscopists [69]. 218 high-quality HRME images from 150 biopsy sites from 130 consecutively enrolled patients were used for this study. 13 endoscopists ( 6 experts and 7 novices) underwent standardized training in HRME image interpretation, following which they were tested on 199 HRME images and asked to give their interpretation and confidence level of image read. Then they were presented with the automated software interpretation and again asked to provide their interpretation and confidence level of image read. Overall, the endoscopists had a pre-software read sensitivity of $84.3 \%$, specificity $75.0 \%$, and accuracy $81.1 \%$ for diagnosis neoplasia. On the post-software read, the endoscopists had a sensitivity of $84.8 \%$, specificity $80.1 \%$, and accuracy of $83.1 \%$. No significant difference in sensitivity, specificity, and accuracy between pre-and post-software read was seen among experts. A significant increase in specificity was seen among novices $(p=0.008)$. When endoscopists had high confidence, there was no significant change in sensitivity and specificity pre- and post-software. Whereas with low confidence, the specificity increased from $58.0 \%$ to $71.0 \%$ $(p=0.004)$ without a significant change in sensitivity. It was demonstrated that an automated diagnosis can be useful for detection and guiding treatment plans, especially among novices. Additionally, preliminary data from a recent study that evaluated the acceptability of HRME among both novice and HRME expert providers suggests that the HRME has a short learning curve, is easy to use, comprehend and overall beneficial in detecting esophageal squamous cell carcinoma [70], (Figure 2).

\section{HRME and Colon cancer}

Parikh, et al. evaluated the use of HRME in differentiating between neoplastic and non-neoplastic colonic lesions in 94 patients undergoing routine colonoscopy [71]. 171 polyps were imaged using WLE followed by HRME and were classified in real time as neoplastic (adenomatous) or non-neoplastic. Results showed that HRME has a significantly higher accuracy, sensitivity and PPV for diagnosing neoplasia as compared to WLE (94\%, $95 \%, 87 \%$ vs. $65 \%, 39 \%$, and $55 \%$ respectively). No difference in NPV was observed.

\section{HRME and Anal cancer}

Varela, et al. explored the role of HRME as an adjunct to high-resolution anoscopy for detecting HighGrade Squamous Intraepithelial Lesions (HSIL) in 41 patients with prior HSIL or abnormal anal cytology [72]. All patients underwent HRME-guided imaging on Lugol's positive areas with subsequent biopsies. After the creation of an image database, 3 expert and 5 novice microendoscopists received training and subsequently were tested on the HRME images. Overall, a high sensitivity of $93 \%$, a specificity of $87 \%$, NPV of $96 \%$ and an accuracy of $89 \%$, for diagnosing HSIL was reported.

In another study by the same group, 62 images from the data based were analyzed to identify quantitative features, that could potentially increase the diagnostic utility of HRME during anoscopy. A total of 11 nuclear features were studied, with a combination of 3 primary measurements yielding a sensitivity of $90 \%$ and a specificity of $88 \%$ for diagnosing HSIL [73].

\section{Summary}

HRME imaging is a comparable but more cost-effective alternative to other imaging modalities such as CLE. Again, interpretation of histopathology like images can be challenging for novice and inexperienced providers, where automation through diagnostic algorithms can prove quite useful. However, the technology, at present, is in clinical trial and not commercially available (Table 3).

\section{Challenges}

As reviewed above, high-resolution endoscopy imaging techniques can allow real-time in vivo histology with high sensitivity and specificity for diagnosing neoplasia in a variety of clinical settings. However, there are several limitations that limit the use of these devices in everyday practice. In this section we will talk about more about those limitations.

\section{Limited field of view}

Subcellular endoscopy can only image a small fraction of the mucosa at a time, and theoretically, it would 

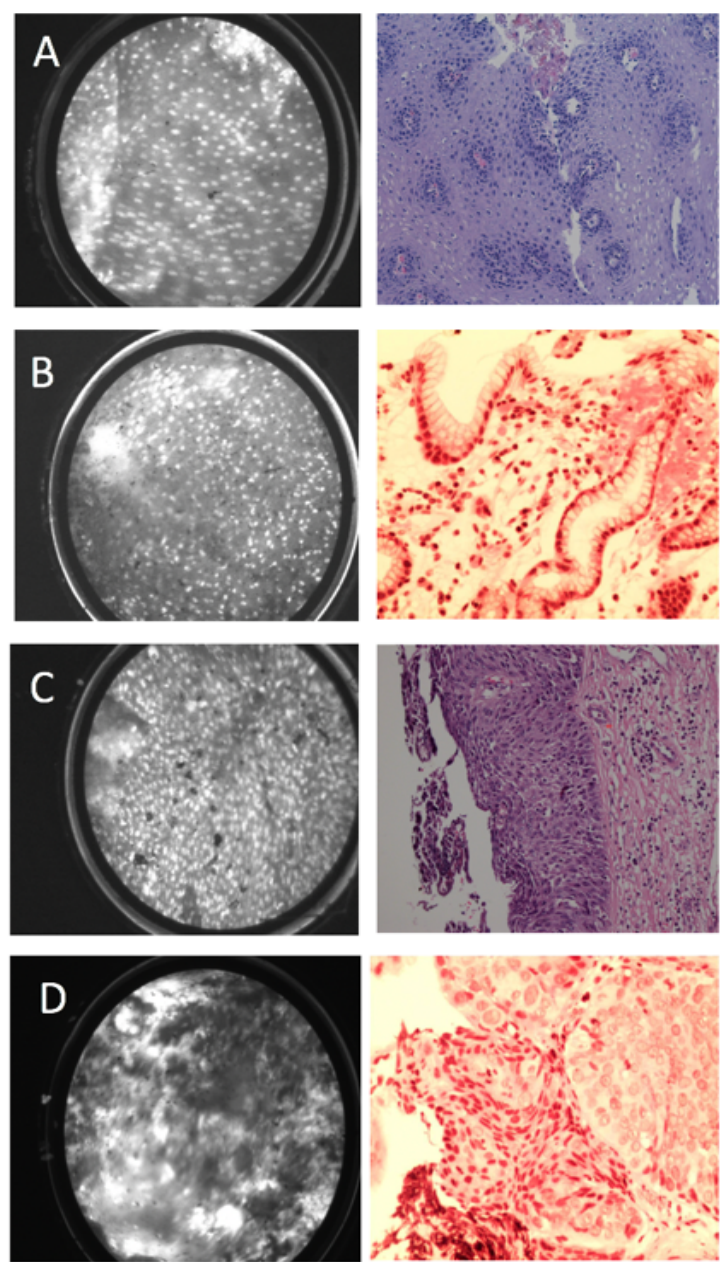

\section{A. Normal squamous epithelium}

Small, round and bright nuclei that are evenly spaced and equally sized nuclei with ample intervening cytoplasm

\section{B. Inflammation}

Nuclei remain small, but are now more crowded with less cytoplasm in between and more irregular spacing

\section{High-Grade Dysplasia}

Nuclei are larger, crowded, and pleomorphic (i.e., exhibiting greater variability in size and shape)

\section{Squamous cell Carcinoma} Large and very pleomorphic nuclei with almost complete loss of cohesion and architectural organization

Figure 2: HRME images and corresponding histopathology for A) Normal squamous epithelium; B) Inflammation; C) Highgrade dysplasia; D) Squamous cell carcinoma.

Table 3: HRME performance.

\begin{tabular}{|l|l|l|l|l|l|}
\hline Reference & Year & Pathology & Sensitivity & Specificity & Accuracy \\
\hline Vila, et al. [33] & 2014 & Esophageal adenocarcinoma & $90 \%$ & $82 \%$ & $90 \%$ \\
\hline Lee, et al. [67] & 2014 & Esophageal adenocarcinoma & $100 \%$ & $94.90 \%$ & $95 \%$ \\
\hline Protano, et al. [34] & 2015 & Esophageal squamous cell carcinoma & $90 \%$ & $89 \%$ & $90 \%$ \\
\hline Shin, et al. [68] & 2015 & Esophageal squamous cell carcinoma & $84-93 \%$ & $92-97 \%$ & $94 \%$ \\
\hline Parikh, et al. [71] & 2015 & Colon cancer & $94 \%$ & $95 \%$ & $94 \%$ \\
\hline Varela, et al. [72] & 2013 & Anal cancer & $90 \%$ & $88 \%$ & \\
\hline
\end{tabular}

take a lot of time to image large sections on mucosa. Newer imaging systems like Nvision VLE system have indeed addressed some of these issues. For other imaging techniques, a potential solution is to pair the high-resolution endoscopy techniques with wide-field image systems such as NBI and chromoendoscopy. These "red flag techniques" can highlight suspicious areas that can then be inspected by these high-resolution endoscopy imaging tools. Video mosaicking is another potential solution, where consecutive video frames are stitched together as the probe moves along the tissue, thereby providing a much larger field of view [74].

\section{Contrast agent}

CLE, EC and HRME all require a contrast agent.
Most commonly used are limited to IV fluorescein, methylene blue, acriflavine or Proflavine. The contrast agents are not targeted or specific and the long-term effects of agents like proflavine are largely unknown. Although these agents have been used in humans without any reported mutagenic, studies in in vivo mouse models have demonstrated some mutagenic potential [75]. A recent study compared rates of cervical neoplasia progression in 392 women in those whose cervical tissue was $(n=232)$ or was not exposed $(n=160)$ to proflavine. There were no significant differences in disease progression from normal/CIN1 to $\mathrm{CIN} 2 / 3$ or from any initial diagnosis to invasive cancer between the proflavine-exposed and control groups overall in nearly two years of fol- 
low up [76]. Overall, there is need for development of more specific, targeted stains and contrast agents.

\section{Learning curve}

Another significant limitation of these techniques is that they all require extensive training and experience. In addition to acquiring good quality, artifact free images, the endoscopist must be trained in interpreting the histopathology like images. Automated diagnostic softwares can help overcome these limitations, however they require further clinical validation.

\section{Cost}

Most of these platforms are quite expensive and can range from $\$ 150,000-\$ 300,000$. While they are invaluable and negate the cost of unnecessary biopsies and pathological interpretations, the initial investment is not feasible in all settings. Hence, they are advantageous in clinical scenarios where a large number of biopsies are performed (BE) and where an immediate diagnosis could alter the treatment plan tremendously (EMR vs. esophagectomy). More affordable solutions like HRME $(<\$ 3600)$ can be used in low-resource settings where more expensive platforms like CLE are not available.

\section{Acceptability}

While these newer technologies have shown exciting results, acceptability among physicians and patients has not yet been evaluated in detail. Since acceptability would directly affect adoption and dissemination of these technologies, future studies that evaluate acceptability are required.

\section{Conclusions}

Newer upcoming imaging modalities are changing the scope of gastrointestinal endoscopy. It has been demonstrated that endoscopy powered by real-time histology and diagnostic algorithms can effectively differentiate neoplastic from non-neoplastic lesions, increase diagnostic yield and also guide treatment plans in the gastrointestinal tract. Combining these new technologies with widefield imaging techniques can facilitate more targeted biopsies to further reduce cost and discomfort associated with unnecessary biopsies and repeat procedures. More affordable alternatives like HMRE can help fill gaps in settings with limited endoscopy and pathology infrastructure. In the future, endoscopy with real-time histology and automated diagnosis could contribute to personalized diagnosis and also increase our understanding of the pathophysiology of various diseases. Further clinical validations and investigations are warranted to assess whether or not these technologies can replace or complement the current standard of care. Although these newer technologies may never be able to completely replace traditional histopathology, they certainly have the potential to optimize and refine endoscopic screening and surveillance.

\section{References}

1. Polglase AL, McLaren WJ, Skinner SA, Kiesslich R, Neurath MF, et al. (2005) A fluorescence confocal endomicroscope for in vivo microscopy of the upper-and the lower-GI tract. Gastrointest Endosc 62: 686-695.

2. Wang TD (2005) Confocal microscopy from the bench to the bedside. Gastrointest Endosc 62: 696-697.

3. Wang TD, Van Dam J (2004) Optical biopsy: A new frontier in endoscopic detection and diagnosis. Clin Gastroenterol Hepatol 2: 744-753.

4. Aisenberg J (2008) Gastrointestinal endoscopy nears "the molecular era". Gastrointest Endosc 68: 528-530.

5. Chauhan SS, Dayyeh BK, Bhat YM, Gottlieb KT, Hwang $\mathrm{JH}$, et al. (2014) Confocal laser endomicroscopy. Gastrointest Endosc 80: 928-938.

6. Kiesslich R, Goetz M, Burg J, Stolte M, Siegel E, et al. (2005) Diagnosing helicobacter pylori in vivo by confocal laser endoscopy. Gastroenterology 128: 2119-2123.

7. Kiesslich R, Burg J, Vieth M, Gnaendiger J, Enders M, et al. (2004) Confocal laser endoscopy for diagnosing intraepithelial neoplasias and colorectal cancer in vivo. Gastroenterology 127: 706-713.

8. Meining A, Frimberger E, Becker V, Von Delius S, Von Weyhern $\mathrm{CH}$, et al. (2008) Detection of cholangiocarcinoma in vivo using miniprobe-based confocal fluorescence microscopy. Clin Gastroenterol Hepatol 6: 1057-1060.

9. Kiesslich R, Goetz M, Vieth M, Galle PR, Neurath MF (2005) Confocal laser endomicroscopy. Gastrointest Endosc Clin N Am 15: 715-731.

10. Wallace MB, Meining A, Canto MI, Fockens $P$, Miehlke S, et al. (2010) The safety of intravenous fluorescein for confocal laser endomicroscopy in the gastrointestinal tract. Aliment Pharmacol Ther 31: 548-552.

11. Chang-Qing Li, Tao Yu, Xiu-Li Zuo, Xiang-Jun Xie, Wen-Bo $\mathrm{Li}$, et al. (2011) Effects on confocal laser endomicroscopy image quality by different acriflavine concentrations. J Interv Gastroenterol 1: 59-63.

12. Neumann H, Kiesslich R, Wallace MB, Neurath MF (2010) Confocal laser endomicroscopy: Technical advances and clinical applications. Gastroenterology 139: 388-392.

13. Dunbar K, Canto M (2008) Confocal endomicroscopy. Curr Opin Gastroenterol 24: 631-637.

14. Goetz M, Malek NP, Kiesslich R (2014) Microscopic imaging in endoscopy: Endomicroscopy and endocytoscopy. Nat Rev Gastroenterol Hepatol 11: 11-18.

15. Kodashima S, Fujishiro M, Takubo K, Kammori M, Nomura $S$, et al. (2006) Ex-vivo study of high-magnification chromoendoscopy in the gastrointestinal tract to determine the optimal staining conditions for endocytoscopy. Endoscopy 38: 1115-1121.

16. Singh R, Sathananthan D, Tam W, Ruszkiewicz A (2013) Endocytoscopy for diagnosis of gastrointestinal neoplasia: The expert's approach. VJEGIE 1: 18-19.

17. Haruhiro Inoue, Tamae Kazawa, Yoshitaka Sato, Hitoshi Satodate, Keita Sasajima, et al. (2004) In vivo observation of living cancer cells in the esophagus, stomach, and colon using catheter-type contact endoscope, "Endo-Cytoscopy system". Gastrointest Endosc Clin N Am 14: 589-594.

18. T Eberl, G Jechart, A Probst, M Golczyk, M Bittinger, et al. (2006) Can an endocytoscope system (ECS) predict histology in neoplastic lesions? Endoscopy 39: 497-501. 
19. Keita Sasajima, Shin-ei Kudo, Haruhiro Inoue, Tsukasa Takeuchi, Hiroshi Kashida, et al. (2006) Real-time in vivo virtual histology of colorectal lesions when using the endocytoscopy system. Gastrointest Endosc 63: 1010-1017.

20. Fercher AF, Hitzenberger CK, Drexler W, Kamp G, Sattmann $\mathrm{H}$ (1993) In vivo optical coherence tomography. Am J Ophthalmol 116: 113-114.

21. Tearney GJ, Brezinski ME, Bouma BE, Boppart SA, Pitris C, et al. (1997) In vivo endoscopic optical biopsy with optical coherence tomography. Science 276: 2037-2039.

22. Fujimoto JG, Brezinski ME, Tearney GJ, Boppart SA, Bouma B, et al. (1995) Optical biopsy and imaging using optical coherence tomography. Nat Med 1: 970-972.

23. Izatt JA, Kulkarni MD, Hsing-Wen W, Kobayashi K, Sivak MV, et al. (1996) Optical coherence tomography and microscopy in gastrointestinal tissues. IEEE 2: 1017-1028.

24. Das A, Sivak MV, Chak A, Wong RCK, Westphal V, et al. (2001) High-resolution endoscopic imaging of the gi tract: A comparative study of optical coherence tomography versus high-frequency catheter probe EUS. Gastrointest Endosc 54: 219-224.

25. Adam Wax, Neil GT, Evan SD, Nicholas JS (2011) Angle-resolved low coherence interferometry for detection of dysplasia in Barrett's esophagus. Gastroenterology 141: 443-447.

26. Yelbuz TM, Choma MA, Thrane L, Kirby ML, Izatt JA (2002) Optical coherence tomography. A new high-resolution imaging technology to study cardiac development in chick embryos. DTUL 106: 2771-2774.

27. Shukla R, Abidi WM, Richards-Kortum R, Anandasabapathy $S$ (2011) Endoscopic imaging: How far are we from real-time histology? World J Gastrointest Endosc 3: 183-194.

28. Leggett CL, Gorospe EC, Chan DK, Muppa P, Owens V, et al. (2016) Comparative diagnostic performance of volumetric laser endomicroscopy and confocal laser endomicroscopy in the detection of dysplasia associated with Barrett's esophagus. Gastrointest Endosc 83: 880-888.

29. Mosko JD, Pleskow D (2017) Evaluation of nine point medical's nvision VLE device for gastrointestinal applications. Expert Rev Med Devices 14: 495-503.

30. Trindade AJ, McKinley M, Fan C, Leggett C, Kahn A, et al. (2019) Endoscopic surveillance of Barret's esophagus using volumetric laser endomicroscopy with artificial intelligence image enhancement. Gastroenterology 157: 303305.

31. Pierce M, Yu D, Richards-Kortum R (2011) High-resolution fiber-optic microendoscopy for in situ cellular imaging. J Vis Exp 47: 2306.

32. Muldoon TJ, Anandasabapathy S, Maru D, Richards-Kortum R (2008) High-resolution imaging in Barrett's esophagus: $A$ novel, low-cost endoscopic microscope. Gastrointest Endosc 68: 737-744.

33. Vila PM, Kingsley MJ, Polydorides AD, Protano MA, Pierce $M C$, et al. (2014) Accuracy and interrater reliability for the diagnosis of barrett's neoplasia among users of a novel, portable high-resolution microendoscope. Dis Esophagus 27: 55-62.

34. Protano MA, Xu H, Wang G, Polydorides AD, Dawsey SM, et al. (2015) Low-cost high-resolution microendoscopy for the detection of esophageal squamous cell neoplasia: An international trial. Gastroenterology 149: 321-329.

35. Levine DS, Haggitt RC, Blount PL, Rabinovitch PS, Rusch
VW, et al. (1993) An endoscopic biopsy protocol can differentiate high-grade dysplasia from early adenocarcinoma in barrett's esophagus. Comparative Study 105: 40-50.

36. Dellon ES, Shaheen NJ (2005) Does Screening for barrett's esophagus and adenocarcinoma of the esophagus prolong survival? J Clin Oncol 23: 4478-4482.

37. Van Sandick JW, Van Lanschot JJ, Kuiken BW, Tytgat GN, Offerhaus GJ, et al. (1998) Impact of endoscopic biopsy surveillance of barrett's oesophagus on pathological stage and clinical outcome of Barrett's carcinoma. Gut 43: 216222.

38. Sharma P, Savides TJ, Canto MI, Corley DA, Falk GW, et al. (2012) The American Society for Gastrointestinal Endoscopy PIVI (preservation and incorporation of valuable endoscopic innovations) on imaging in barrett's esophagus. Gastrointest Endosc 76: 252-254.

39. Siegel RL, Miller KD, Jemal A (2019) Cancer statistics. CA Cancer J Clin 69: 7-34.

40. Canto MI, Anandasabapathy S, Brugge W, Falk GW, Dunbar KB, et al. (2014) In vivo endomicroscopy improves detection of barrett's esophagus-related neoplasia: A multicenter international randomized controlled trial (with video). Gastrointest Endosc 79: 211-221.

41. Sharma $P$, Meining AR, Coron E, Lightdale CJ, Wolfsen $\mathrm{HC}$, et al. (2011) Real-time increased detection of neoplastic tissue in barrett's esophagus with probe-based confocal laser endomicroscopy: Final results of an international multicenter, prospective, randomized, controlled trial. Gastrointest Endosc 74: 465-472.

42. Pech $\mathrm{O}$, Rabenstein $\mathrm{T}$, Manner $\mathrm{H}$, Petrone MC, Pohl J, et al. (2008) Confocal laser endomicroscopy for in vivo diagnosis of early squamous cell carcinoma in the esophagus. Clin Gastroenterol Hepatol 6: 89-94.

43. Huang J, Yang YS, Lu ZS, Wang SF, Yang J, et al. (2015) Detection of superficial esophageal squamous cell neoplasia by chromoendoscopy-guided confocal laser endomicroscopy. World J Gastroenterol 21: 6974-6981.

44. Kitabatake S, Niwa Y, Miyahara R, Ohashi A, Matsuura T, et al. (2006) Confocal endomicroscopy for the diagnosis of gastric cancer in vivo. Endoscopy 38: 1110-1114.

45. Jeon SR, Cho WY, Jin SY, Cheon YK, Choi SR, et al. (2011) Optical biopsies by confocal endomicroscopy prevent additive endoscopic biopsies before endoscopic submucosal dissection in gastric epithelial neoplasias: A prospective, comparative study. Gastrointest Endosc 74: 772-780.

46. Bok GH, Jeon SR, Cho JY, Cho JH, Lee WC, et al. (2013) The accuracy of probe-based confocal endomicroscopy versus conventional endoscopic biopsies for the diagnosis of superficial gastric neoplasia (with videos). Gastrointest Endosc 77: 899-908.

47. Shahid MW, Buchner AM, Heckman MG, Krishna M, Raimondo M, et al. (2012) Diagnostic accuracy of probe-based confocal laser endomicroscopy and narrow band imaging for small colorectal polyps: A feasibility study. 107: 231-239.

48. Shahid MW, Buchner AM, Coron E, Woodward TA, Raimondo M, et al. (2012) Diagnostic accuracy of probe-based confocal laser endomicroscopy in detecting residual colorectal neoplasia after EMR: A prospective study. Gastrointest Endosc 75: 525-533.

49. Wallace M, Lauwers GY, Chen Y, Dekker E, Fockens P, et al. (2011) Miami classification for probe-based confocal laser endomicroscopy. Endoscopy 43: 882-891. 
50. Pohl H, Koch M, Khalifa A, Papanikolaou IS, Scheiner K, et al. (2007) Evaluation of endocytoscopy in the surveillance of patients with barrett's esophagus. Endoscopy 39: 492496.

51. Eberl T, Jechart G, Probst A, Golczyk M, Bittinger $M$, et al. (2007) Can an endocytoscope system (ECS) predictin neoplastic lesions? Endoscopy 39: 497-501.

52. Inoue H, Sasajima K, Kaga M, Sugaya S, Sato $Y$, et al. (2006) Endoscopic in vivo evaluation of tissue atypia in the esophagus using a newly designed integrated endocytoscope: A pilot trial. Endoscopy 38: 891-895.

53. Kaise $M$, Ohkura $Y$, lizuka $T$, Kimura R, Nomura $K$, et al. (2015) Endocytoscopy is a promising modality with high diagnostic accuracy for gastric cancer. Endoscopy 47: 19-25.

54. Mori Y, Kudo S, Ikehara N, Wakamura K, Wada Y, et al. (2013) Comprehensive diagnostic ability of endocytoscopy compared with biopsy for colorectal neoplasms: A prospective randomized noninferiority trial. Endoscopy 45: 98-105.

55. Takeda K, Kudo SE, Mori Y, Misawa M, Kudo T, et al. (2017) Accuracy of diagnosing invasive colorectal cancer using computer-aided endocytoscopy. Endoscopy 49: 798802.

56. Poneros JM, Brand S, Bouma BE, Tearney GJ, Compton CC, et al. (2001) Diagnosis of specialized intestinal metaplasia by optical coherence tomography. Gastroenterology 120: 7-12.

57. Evans JA, Poneros JM, Bouma BE, Bressner J, Halpern $E F$, et al. (2006) Optical coherence tomography to identify intramucosal carcinoma and high-grade dysplasia in barrett's esophagus. Clin Gastroenterol Hepatol 4: 38-43.

58. Evans JA, Bouma BE, Bressner J, Shishkov M, Lauwers GY, et al. (2007) Identifying intestinal metaplasia at the squamocolumnar junction by using optical coherence tomography. Gastrointest Endosc 65: 50-56.

59. Smith MS, Cash B, Konda V, Trindade AJ, Gordon S, et al (2019) Volumetric laser endomicroscopy and its application to barrett's esophagus: Results from a 1,000 patient registry. Dis Esophagus 32.

60. Swager AF, Tearney GJ, Leggett CL, Van Oijen MGH, Meijer SL, et al. (2019) Identification of volumetric laser endomicroscopy features predictive for early neoplasia in barrett's esophagus using high-quality histological correlation. Gastrointest Endosc 85: 918-926.

61. Hatta W, Uno K, Koike T, Yokosawa S, lijima K, et al. (2010) Optical coherence tomography for the staging of tumor infiltration in superficial esophageal squamous cell carcinoma. Gastrointest Endosc 71: 899-906.

62. Hatta W, Uno K, Koike T, lijima K, Asano N, et al. (2012) A prospective comparative study of optical coherence tomography and EUS for tumor staging of superficial esophageal squamous cell carcinoma. Gastrointest Endosc 76: 548-555.

63. Trindade AJ, Benias PC, Inamdar S, Fan C, Sethi A, et al. (2018) Use of volumetric laser endomicroscopy for determining candidates for endoscopic therapy in superficial esophageal squamous cell carcinoma. United European Gastroenterol J 6: 838-845.
64. Jäckle S, Gladkova N, Feldchtein F, Terentieva A, Brand $B$, et al. (2000) In vivo endoscopic optical coherence tomography of the human gastrointestinal tract-toward optical biopsy. Endoscopy 32: 743-749.

65. Pfau PR, Sivak MV, Chak A, Kinnard M, Wong RCK, et al. (2003) Criteria for the diagnosis of dysplasia by endoscopic optical coherence tomography. Gastrointest Endosc 58: 196-202.

66. Trindade AJ, Sultan K, Vamadevan AS, Fan C, Sejpal DV (2016) Successful use of volumetric laser endomicroscopy in imaging a rectal polyp. Therap Adv Gastroenterol 9: 128131.

67. Lee MH, Parikh N, Polydorides AD, Louie J, Protano MA, et al. (2014) Su2011 diagnostic yield and clinical impact of a low-cost microendoscope in the early diagnosis of Barrett's associated neoplasia: A prospective, single-center randomized controlled trial. Gastroenterology 146: 522.

68. Shin D, Protano MA, Polydorides AD, Dawsey SM, Pierce MC, et al. (2015) Quantitative analysis of high-resolution microendoscopic images for diagnosis of esophageal squamous cell carcinoma. Clin Gastroenterol Hepatol 13: 272279.

69. Tan MC, Bhushan SR, Patel K, Hammad T, Khan A, et al. (2019) Tu1960 using artificial intelligence in diagnosis of esophageal squamous cell neoplasia: A post-hoc study of high-resolution microendoscopy (HRME) image interpretation. Gastrointest Endosc 89: 632-633.

70. Chen H, Wu C, Bhushan SR, Zhan T, Liu C, et al. (2019) Sa1027-Beyond availability: Evaluating acceptability of high resolution microendoscopy among patients and providers for detection of esophageal squamous cell neoplasia. Gastroenterology 156: 260.

71. Parikh ND, Perl D, Lee MH, Chang SS, Polydorides AD, et al. (2015) In vivo classification of colorectal neoplasia using high-resolution microendoscopy: Improvement with experience. J Gastroenterol Hepatol 30: 1155-1160.

72. Varela BR, Perl D, Zhou E, Chang SS, Protano MA, et al. (2013) Mo1645 high-resolution microendoscopy (HRME) in the detection of anal intraepithelial neoplasia: Assessment of accuracy and interobserver variability. Gastrointest Endosc 77: 456-457.

73. Varela BR, Shin D, Perl D, Zhou E, Lee MH, et al. (2014) Sa1168 High-resolution microendoscopy (HRME) in the detection of anal intraepithelial neoplasia: A quantitative approach to optimize diagnostic accuracy. Gastroenterology 146: 218.

74. Bedard N, Quang T, Schmeler K, Richards-Kortum R, Tkaczyk TS (2012) Real-time video mosaicing with a high-resolution microendoscope. Biomed Opt Express 3: 24282435.

75. Speck WT, Rosenkranz HS (1980) Proflavin: An unusual mutagen. Mutat Res 77: 37-43.

76. Pantano N, Hunt B, Schwarz RA, Parra S, Cherry K, et al. (2018) Is proflavine exposure associated with disease progression in women with cervical dysplasia? A brief report. Photochem Photobiol 94: 1308-1313. 